\title{
Recent advances in understanding Golgi biogenesis
}

\section{Suzanne R Pfeffer}

Address: Department of Biochemistry, Stanford University School of Medicine, Stanford, CA 94305-5307, USA

Email: pfeffer@stanford.edu

FI000 Biology Reports 2010, 2:32 (doi:10.34I0/B2-32)

The electronic version of this article is the complete one and can be found at: http://fl000.com/reports/biology/content/2/32

\begin{abstract}
The Golgi complex is a central processing station for proteins traversing the secretory pathway, yet we are still learning how this compartment is constructed and how cargo moves through it. Recent experiments suggest a key role for Ras-like Rab GTPases and provide important new ideas for how the Golgi may function.
\end{abstract}

\section{Introduction and context}

After their biosynthesis at the endoplasmic reticulum, secreted proteins are transported to the Golgi complex, where they are post-translationally modified and sorted for secretion, plasma membrane delivery, or delivery to prelysosomes. The Golgi contains multiple subcompartments, termed cis (early), medial (middle), and trans (late) cisternae; each of these subcompartments houses different sets of glycosyltransferases and other enzymes. Proteins enter the Golgi at the cis compartment and exit at the trans compartment, but how they move from one cisterna to the next is still being determined.

Two possible models are widely discussed [1-3]. According to the cisternal maturation (or progression) model, cargo remains in a given compartment and different enzymes arrive there to convert a cis cisterna into a medial one or a medial cisterna into a trans cisterna. This type of maturation is widely accepted in the endocytic pathway [4]. Alternatively, cargo moves from one Golgi compartment to the next, encountering different enzymes in each subsequent compartment until it reaches the trans cisterna, where it is then sorted into carriers bound for post-Golgi destinations. This second class of model could use vesicles to transport cargo from one compartment to the next or compartmentconnecting tubules through which cargo could pass.

Cisternal maturation has been visualized directly in yeast: two groups have detected the 'conversion' of one Golgi compartment into another by high-resolution, live cell video microscopy [5,6]. A limitation of those studies is that one of the compartment markers that was monitored is a peripheral membrane protein that is likely to reversibly bind to and release from the Golgi surface. Also, it has not yet been possible to visualize cargo simultaneously.

The situation may be more complex in mammalian cells, where Golgi cisternae are stacked tightly together, unlike yeast; it is hard to imagine a single cisterna moving from one side of the well-stacked structure to the other. Nevertheless, large procollagen cargo traverses the Golgi without ever leaving a cisterna [7], in support of a maturation model. To complicate matters, membrane tubules have been detected between Golgi cisternae under conditions of active secretion [8]; this scenario would permit cargo movement from one side of the stack to the next without maturation or vesicle transfer.

\section{Major recent advances}

Important new clues to how Golgi compartments might 'mature' come from a study of Golgi-localized, Ras-related, Rab family GTPases in yeast. Rab GTPases are localized to different membrane compartments and catalyze the formation of functionally distinct, membrane microdomains that are important for transport vesicle formation, vesicle motility, and vesicle (or compartment) docking and fusion [9].

Rab GTPases help early endosomes mature into later endosomes by a process called Rab conversion [4]. The 
early endosomal Rab5 protein recruits a specific guanine nucleotide exchange factor (GEF) that activates Rab7. Rab7 then recruits Rab7-specific effectors to that compartment, thereby converting an early endosome into a late endosome. This type of Rab cascade (Figure 1B) was first described for a yeast Golgi Rab, Ypt32p, recruiting the GEF for the subsequent acting Sec4p Rab [10].

Rivera-Molina and Novick [11] have now used live cell video microscopy to detect Rab conversion at the yeast Golgi: they see compartments containing the early Golgi Rab, Ypt1p, convert into a compartment containing the late Golgi Rab, Ypt32p. (Although the light microscopy method employed could not resolve structures smaller than about $200 \mathrm{~nm}$, the images were nevertheless highly compelling.) The process involves the recruitment of Ypt32p by the GTPase-activating protein (GAP) that inactivates Ypt1p: Gyp1p. Upon inactivation, Ypt1p becomes a substrate for removal from membranes by another protein, GDI (GDP-dissociation inhibitor). The removal of Rabs from the membranes makes this work subject to one of the same limitations of the previous studies [5,6]; nevertheless, these markers permitted the authors to detect an important molecular transformation. The data provide a direct molecular mechanism for compartment inter-conversion at the Golgi, reminiscent of maturation in the endocytic pathway. Very importantly, the authors wrote that in addition to compartment conversion, "... close examination suggests that other processes may contribute as well. Golgi compartments were seen to be dynamic, undergoing a certain amount of fission and fusion. In some cases (30\%), a Ypt32p compartment appeared to fuse to a Ypt1p compartment to yield a mixed compartment or a mixed compartment appeared to undergo segregation and fission to yield separate Ypt1p and Ypt32p compartments" [11]. What this means is that, yes, yeast Golgi compartments undergo apparent maturation by Rab conversion, and at the same time, cargo may get the 'fast track' from one compartment to the next by intermittent cisternal fusion and fission events (Figure 2A,B). Importantly, although a compartment will seem to mature, it is actually forming from a stable predecessor.

The ability of Golgi cisternae to undergo fission and fusion has been known since the 1970s: simple, nocodazoletriggered depolymerization of microtubules causes the mammalian Golgi to fragment into mini-stacks that disperse throughout the cytoplasm, and drug washout leads to rapid stack reassembly (Figure 2A). This indicates that the Golgi is capable of fission as soon as microtubules are lost and of fusion with itself as soon as microtubules repolymerize. Compartment collisions likely enhance
Figure I. Compartment maturation by fission/fusion and Rab conversion

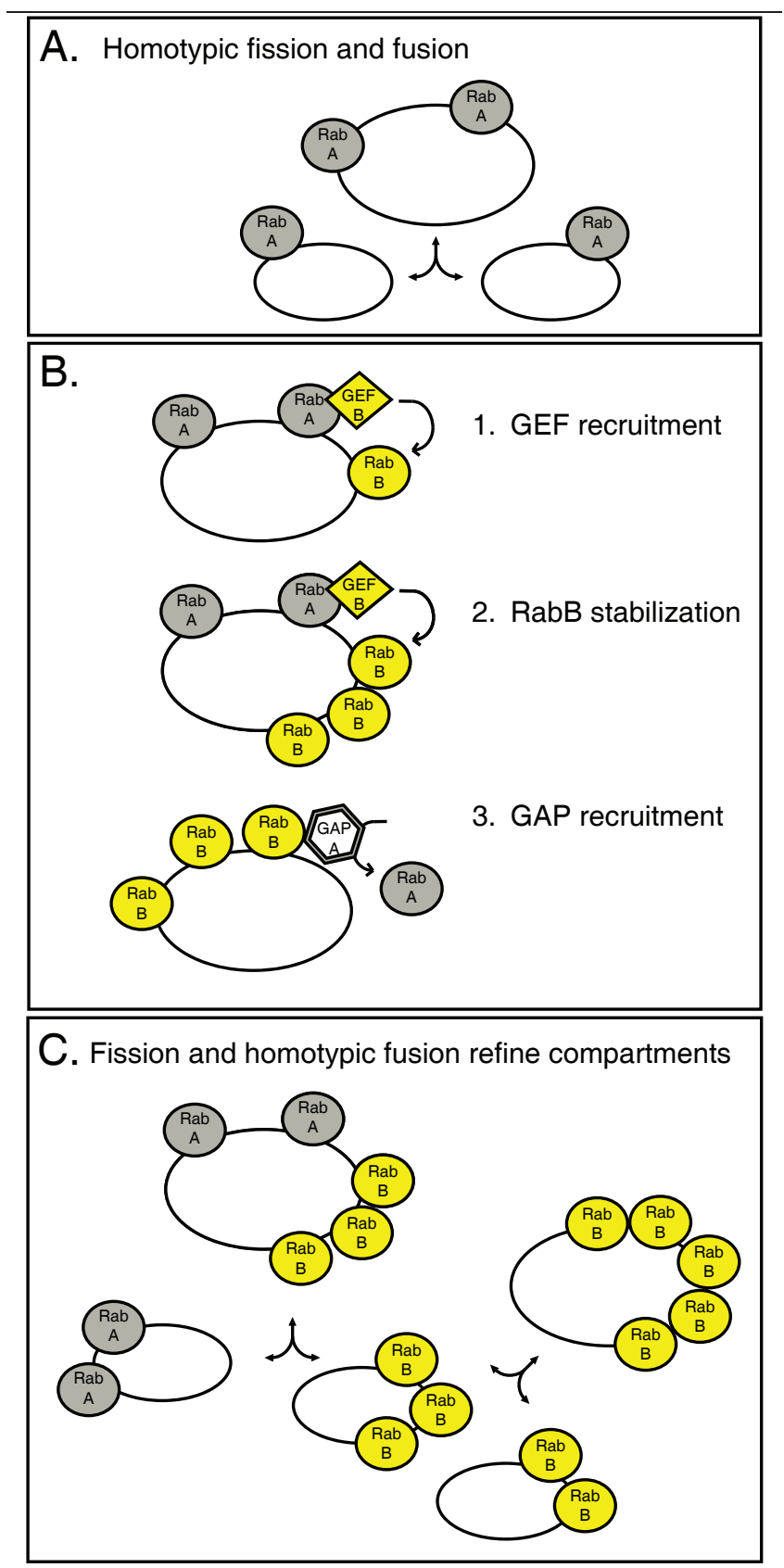

(A) Endoplasmic reticulum, Golgi, and endosome membranes are capable of homotypic fusion and fission. Fusion is driven by Rab GTPases that recruit docking and fusion proteins. Fission often requires microtubules and motor proteins. (B) Rab cascades occur when sequentially acting Rabs recruit guanine nucleotide exchange factors (GEFs) and GTPase-activating proteins (GAPs) to membranes. RabA recruits a GEF that will convert the subsequent acting Rab to its active form. GTP-Rabs are stabilized on membranes by effector binding. RabB can then recruit a GAP that will inactive the previous acting Rab, thereby removing it from the newly formed, second compartment. (C) Mixed compartment fission can also segregate early and later compartments. This can be refined by GAPs as in (B). 
Figure 2. Homotypic fusion could permit large cargo to traverse the stack without cisternal maturation

A. Golgi stack fission and fusion

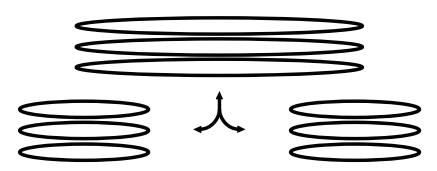

B. Rab cascades create sequential domains that can undergo homotypic fusion and fission

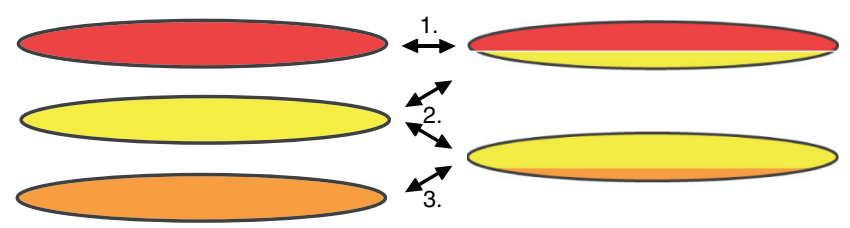

(A) Golgi stacks also undergo homotypic fusion and fission. Early Golgi has the capacity to fuse with other early Golgi cisternae and so on. Fission is favored in the absence of microtubules or when Golgin proteins are depleted from cells. (B) Large cargo could use homotypic fusion to move through the stack. Glycosyltransferases are localized to cis (red), medial (yellow), and trans (orange) Golgi compartments but rarely have a perfectly sharp distribution. Colors represent the predicted location of specific Rab domains. Thus, for example, cis-Golgi homotypic fusion events can occur with another cis compartment (step I) or perhaps a medial (yellow) compartment (or both). Similarly, a medial cisterna may be able to fuse with a trans cisterna (orange). In this manner, a large cargo may be able to encounter all Golgi-processing enzymes without entering a transport vesicle, indicated by fusion steps (arrows) I, 2, and then 3 .

fusion, and cytoskeletal motor proteins that decorate the Golgi and connect it to both microtubules and actin cables are sure to contribute to both fusion and fission events, as is true in the endocytic pathway (Figure 1A,C).

Are intercisternal fusion/fission connections required for membrane traffic? Transport is only partially blocked in nocodazole-treated cells and this condition favors stack fission. But homotypic fission and fusion are likely much more prevalent than previously anticipated because cellular depletion of any one of many different Golgi proteins ('Golgins') generates mini-stacks that are clustered near the microtubule-organizing center $[12,13]$. Such transient fusion and fission could yield the tubules that have been detected in electron micrographs of mammalian cell Golgi complexes [8]. Fission and fusion would make it possible to accommodate extra-large cargoes, such as collagen, that are too big to fit into conventional transport vesicles.

\section{Future directions}

These data support a new model for transport through the Golgi. As is well established for the endocytic pathway, Rab GTPases would define specific subdomains and retain specific Golgi enzyme subsets there. Compartments would be defined by their distinct Rab GTPases, and adjacent cisternae might fuse at some frequency that allows cargoes to encounter sequentially acting, processing enzymes. In a mixed compartment, Rab GEFs and GAPs would segregate individual Rabs into separate regions that would be segregated upon the simple action of a membrane-associated, cytoskeletal motor protein to drive fission. Order within the stack would be maintained by the relationship between specific Rabs and their cognate activators (GEFs) and inactivators (GAPs), which are designed to permit Rabs to function in a sequential cascade. Indeed, the proteins that stack the cisternae may use a Rab cascade to achieve their position in the stack [14]. At the trans Golgi, Rabs would also initiate the process by which specific cargoes are collected into distinct transport carriers and delivered to their final destinations.

Validation and clarification of this model will require defining which Rabs build which specific Golgi enzyme microdomains and determining the specific molecular interactions that permit fission, fusion, and enzyme organization. Vesicles are likely to be involved in Golgi transport: we know that COP-I-coated vesicles collect KDEL receptors for delivery back to the endoplasmic reticulum; in this case, we can postulate that such vesicles bud from a Rab GTPase-organized, functional membrane microdomain. The same proteins that drive vesicle targeting and fusion may also participate in cisternal docking and fusion to permit protein transport across this central cellular compartment. The mechanism by which depletion of any one of at least 10 different Golgin proteins leads to mini-stack formation will likely tell us much about how proteins move through the mammalian Golgi stack. Additional analysis of Golgi transport vesicles will also help clarify transport through the Golgi.

\section{Abbreviations}

GAP, GTPase-activating protein; GEF, guanine nucleotide exchange factor.

\section{Competing interests}

The author declares that she has no competing interests.

\section{Acknowledgments}

Research in the author's lab is supported by the National Institutes of Health (DK37332 and GM 079322).

\section{References}

I. Emr S, Glick BS, Linstedt AD, Lippincott-Schwartz J, Luini A, Malhotra V, Marsh BJ, Nakano A, Pfeffer SR, Rabouille C, Rothman JE, Warren G, Wieland FT: Journeys through the Golgi-taking stock in a new era. J Cell Biol 2009, 187:449-53. 
2. Glick BS, Nakano A: Membrane traffic within the Golgi apparatus. Annu Rev Cell Dev Biol 2009, 25: I I3-32.

3. Pelham HR, Rothman JE: The debate about transport in the Golgi-two sides of the same coin? Cell 2000, 102:713-9.

4. Rink J, Ghigo E, Kalaidzidis $Y$, Zerial M: Rab conversion as a mechanism of progression from early to late endosomes. Cell 2005, 1 22:735-49.

FI000 Factor 8.0 Exceptional

Evaluated by Vojo Deretic 30 Sep 2005, David Bilder 05 Jan 2006

5. Losev E, Reinke CA, Jellen J, Strongin DE, Bevis BJ, Glick BS: Golgi maturation visualized in living yeast. Nature 2006, 44I: I002-6.

FI000 Factor 10.I Exceptional

Evaluated by Tom Stevens 30 May 2006, David Stephens 05 Jun 2006, Davis Ng 20 Jun 2006, Catherine Jackson 12 Jul 2006, Viki Allan 28 Jul 2006

6. Matsuura-Tokita K, Takeuchi M, Ichihara A, Mikuriya K, Nakano A: Live imaging of yeast Golgi cisternal maturation. Nature 2006, 44I: $1007-10$.

FI000 Factor 10.5 Exceptional

Evaluated by Tom Stevens 30 May 2006, Antonella De Matteis 02 Jun 2006, David Stephens 05 Jun 2006, Davis Ng 20 Jun 2006 Catherine Jackson II Jul 2006, Viki Allan 28 Jul 2006

7. Bonfanti L, Mironov AA Jr, Martínez-Menárguez JA, Martella $O$, Fusella A, Baldassarre M, Buccione R, Geuze HJ, Mironov AA, Luini A: Procollagen traverses the Golgi stack without leaving the lumen of cisternae: evidence for cisternal maturation. Cell 1998, 95:993-1003.

8. Trucco A, Polishchuk RS, Martella O, Di Pentima A, Fusella A, Di Giandomenico D, San Pietro E, Beznoussenko GV, Polishchuk EV, Baldassarre M, Buccione R, Geerts WJ, Koster AJ, Burger KN,
Mironov AA, Luini A: Secretory traffic triggers the formation of tubular continuities across Golgi sub-compartments. Nat Cell Biol 2004, 6:|07|-8|.

FI000 Factor 6.4 Must Read

Evaluated by Paul Gleeson 09 Nov 2004, Robert Parton 23 Nov 2004

9. Grosshans BL, Ortiz D, Novick P: Rabs and their effectors: achieving specificity in membrane traffic. Proc Natl Acad Sci U S A 2006, I03:| | $82 \mid-7$.

10. Ortiz D, Medkova M, Walch-Solimena C, Novick P: Ypt32 recruits the Sec4p guanine nucleotide exchange factor, Sec2p, to secretory vesicles; evidence for a Rab cascade in yeast. J Cell Biol 2002, I 57:1005-I5.

FI000 Factor 6.4 Must Read

Evaluated by Francis Barr 12 Sep 2002, Catherine Jackson 31 Jan 2003

II. Rivera-Molina FE, Novick PJ: A Rab GAP cascade defines the boundary between two Rab GTPases on the secretory pathway. Proc Natl Acad Sci U S A 2009, 106:14408-13.

12. Reddy JV, Burguete AS, Sridevi K, Ganley IG, Nottingham RM, Pfeffer SR: A functional role for the GCCI 85 golgin in mannose 6-phosphate receptor recycling. Mol Biol Cell 2006, 17:4353-63.

13. Puthenveedu MA, Bachert C, Puri S, Lanni F, Linstedt AD: GMI30 and GRASP65-dependent lateral cisternal fusion allows uniform Golgi-enzyme distribution. Nat Cell Biol 2006, 8:238-48.

FI000 Factor 3.0 Recommended

Evaluated by Catherine Rabouille 07 Mar 2006

14. Pfeffer SR: Constructing a Golgi complex. J Cell Biol 200I, I55:873-5. 